Dr. med.

Brigitte Moreano

Stellvertretende

Chefredakteurin

brigitte.moreano@

springer.com
Früher Therapiebeginn wäre entscheidend, aber:

\section{Wegen RA-Symptomen gehen viele nicht zum Arzt}

Symptome einer beginnenden rheumatoiden Arthritis (RA) werden oft nicht ernst genommen, wie eine britische Studie nahelegt. Die Autoren hatten Patienten aus Allgemeinarztpraxen befragt, wie sie bei entsprechenden Symptomen reagieren würden. Neben RA-Vignetten (Gelenkschmerzen, Steifheit, Schwellung) wurden den Teilnehmern auch Darmkrebs- und Angina-pectoris-Symptome zur Beurteilung vorgelegt. Insgesamt hielten die über
1.000 Befragten die RA-Symptome nicht für besonders beunruhigend. Mit dem Arztbesuch würden die meisten zumindest einige Wochen abwarten; nicht selten war man sogar bereit, „mit den Beschwerden zu leben“. Die Symptome von Darmkrebs und Angina pectoris wurden dagegen wesentlich besser erkannt und die Bereitschaft, deshalb zum Arzt zu gehen, war deutlich größer.

- Arthr Care Res 2017; online 10. April; doi: 10.1002/acr.22979

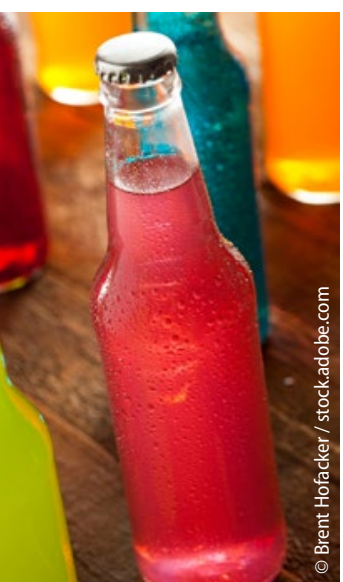

\title{
Fragliche Kausalbeziehung
} \section{Sind gesüßte Softdrinks schlecht fürs Hirn?}

Der Konsum von Softdrinks mit künstlichen Süßstoffen geht mit einem höheren Risiko für Schlaganfälle und Demenz einher, wie eine Studie aus den USA ergeben hat. Knapp 3.000 Probanden waren regelmäßig zu ihren Trinkgewohnheiten befragt worden. 97 Teilnehmer erlitten einen Schlaganfall, 81 erkrankten an einer Demenz. Der Konsum von künstlich gesüßten Softdrinks war signifikant mit beiden Erkrankungen assoziiert. Wer bis zu sieben Jahre lang mindestens einmal täglich zu solchen Getränken griff, hatte ein 2,6-fach erhöhtes Schlaganfallrisiko und ein 2,89-fach höheres Risiko, an M. Alzheimer zu erkranken.

- Stroke 2017;online 24. April

\section{Lange Arbeitszeiten erhöhen kardiovaskuläres Risiko}

\section{Ab 52 Wochenstunden wird es kritisch}

Eine lange Wochenarbeitszeit erhöht das Risiko für kardiovaskuläre Erkrankungen und Krebs, so das Ergebnis einer Studie der Universität von Texas. Die Autoren stützten sich dabei auf eine Längsschnittstudie (PSID), die Daten von mehr als 18.000 Menschen dokumentiert. Berücksichtigt wurden Teilnehmer, für die Daten zur Arbeitszeit über mindestens zehn Jahre vorlagen. Die Wissenschaftler errechneten einen Grenzwert von

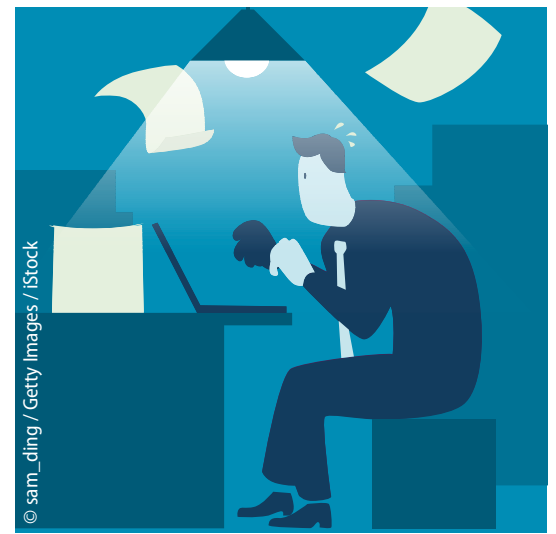

52 Wochenstunden, ab dem das Risiko für kardiovaskuläre Erkrankungen oder Krebs signifikant erhöht ist. Im Vergleich zu Menschen mit 35-51 Wochenarbeitsstunden war das Risiko für einen schlechten Gesundheitszustand um $28 \%$ und das für kardiovaskuläre Erkrankungen und Krebs um 42\% bzw. $62 \%$ erhöht.

- Am J Epidemiol 2017; online 28. April. doi: 10.1093/aje/ $k w \times 003$ 